\title{
Toxicidades orais da terapia oncológica em crianças e adolescentes: um estudo descritivo
}

\section{Oral toxicities of oncology therapy in children and adolescents: a descriptive study}

\section{Toxicidades orales de la terapia contra el cáncer en niños y adolescentes: un estudio descriptivo}

Lísia Daltro Borges Alves ${ }^{1 凶} \underline{\text { ORCID }}$, Isabela Sampaio Cerqueira ${ }^{1 凶} \underline{\text { ORCID }}$, Franco Arsati ${ }^{2} \underline{\text { ORCID }}$, Alessandra Laís Pinho Valente-Pires ${ }^{3 凶} \underline{\text { ORCID }}$, Valéria Souza Freitas $4 \llbracket \underline{\text { ORCID }}$

\footnotetext{
${ }^{1}$ Cirurgiã-dentista, Departamento de Saúde, Universidade Estadual de Feira de Santana (UEFS), Bahia, Brasil.

2 Doutor em Odontologia pela Universidade Estadual de Campinas (UNICAMP), Professor Adjunto, Departamento de Ciências Biológicas, Universidade Estadual de Feira de Santana (UEFS), Bahia, Brasil.

${ }^{3}$ Mestre em Saúde Coletiva, Departamento de Saúde, Universidade Estadual de Feira de Santana (UEFS), Bahia, Brasil.

${ }^{4}$ Doutora em Patologia Oral pela Universidade Federal do Rio Grande do Norte, Professora Adjunta, Departamento de Saúde, Universidade Estadual de Feira de Santana (UEFS), Bahia, Brasil.
}

Fecha correspondencia: Recibido: octubre de 2020. Aprobado: abril de 2021.

Forma de citar:

Borges Alves LD, Sampaio Cerqueira I, Arsati F, Pinho Valente Pires AL, Souza Freitas V. Toxicidades orais da terapia oncológica em crianças e adolescentes: um estudo descritivo. Rev. CES Odont 2021; 34(2): 30-45.

https://doi.org/10.21615/ cesodon.5887

\section{Open access}

(C) Derecho de autor

Licencia creative commons

Ética de publicaciones

Revisión por pares

Gestión por Open Journal System

\section{Resumen}

Introducción y objetivo: El cáncer es la segunda causa de muerte entre niños y adolescentes en Brasil. Evaluar la prevalencia de toxicidades orales derivadas del tratamiento oncológico en niños y adolescentes atendidos en el Hospital Estadual da Criança, de 2016 a 2017. Materiales y métodos: Se trata de un estudio descriptivo con datos obtenidos de historias clínicas. Se estudiaron variables relacionadas con la neoplasia, el portador, las toxicidades orales y el tratamiento oncológico, las cuales fueron analizadas de forma descriptiva y se realizó la prueba de la $\chi 2$ de Fisher para algunas de ellas. Resultados: Se identificaron 58 pacientes, en su mayoría mujeres (53\%) y con edades entre 3 y 5 años $(28 \%)$, con una media de 6,62 años. La neoplasia más común fue la leucemia linfoide aguda (38\%), siendo la quimioterapia sola, la modalidad terapéutica más utilizada (67\%), especialmente con el uso de citarabina (60\%) y vincristina (56\%). Veintitrés (40\%) de los niños y adolescentes presentaron alguna toxicidad oral 
DOI: $10.21615 /$ cesodon.5887 ISSNe 2215-9185 ISSN 0120-971X

Publica con nosotros durante la terapia oncológica, por lo que a $4(17,4 \%)$ se les interrumpió el tratamiento antineoplásico. La mucositis oral (70\%) fue la toxicidad más común, afectando los labios en el $55 \%$ y fue asociada a dolor en el $64 \%$ de los casos. Dieciocho $(78,2 \%)$ pacientes recibieron tratamiento por toxicidad oral, prescribiéndose nistatina en el $88,9 \%$ de los casos. Además,

hubo una asociación significativa entre el estado de supervivencia y el lugar de residencia $(p=0,03)$. Conclusiones: Entre los 58 niños y adolescentes estudiados, el $40 \%$ presentó toxicidades orales y 4 tuvieron interrumpido el tratamiento antineoplásico por este motivo. Así, se refuerza la importancia y la necesidad de la actuación del odontólogo en el equipo multidisciplinario en oncología.

Palabras clave: manifestaciones bucales; protocolos antineoplásicos; estomatitis; grupo de atención al paciente.

\section{Abstract}

Introduction and objective: Cancer is the second leading cause of death among children and adolescents in Brazil. To evaluate the prevalence of oral toxicities resulting from cancer treatment in children and adolescents treated at the Hospital Estadual da Criança, from 2016 to 2017. Material and methods: This is a descriptive study with data obtained from medical records. Variables related to neoplasia, carrier, oral toxicities and cancer treatment were studied, which were analyzed descriptively and thFisher $\chi 2$ test was performed for some of them. Results: Fifty eigth patients were identified, mostly female (53\%) and aged between 3 and 5 years (28\%), with an average of 6.62 years. The most frequent neoplasm was acute lymphoid leukemia (38\%), with chemotherapy alone being the most used therapeutic modality (67\%), especially with the use of cytarabine (60\%) and vincristine (56\%). Twenty-three (40\%) of the children and adolescents exhibited some oral toxicity during cancer therapy, and for this reason, $4(17.4 \%)$ had their antineoplastic treatment interrupted. Oral mucositis (70\%) was the most common toxicity, affecting the lips in $55 \%$ and being associated with pain in $64 \%$ of cases. Eighteen (78.2\%) patients received treatment for oral toxicities, with nystatin being prescribed in $88.9 \%$ of cases. In addition, there was a significant association between survival status and place of residence ( $p=0.03$ ). Conclusions: Among the 58 children and adolescents studied, $40 \%$ had oral toxicities and 4 had their antineoplastic treatment interrupted for this reason. Thus, the importance and the need for the performance of the dentist in the multidisciplinary team in oncology is reinforced. 
Keywords: oral manifestations; antineoplastic protocols; stomatitis; patient care team.

\section{Resumo}

Introdução e objetivo: O câncer é a segunda maior causa de morte entre crianças e adolescentes no Brasil. Avaliar a prevalência de toxicidades orais decorrentes do tratamento oncológico em crianças e adolescentes atendidos no Hospital Estadual da Criança, no período de 2016 a 2017. Materiais e métodos: Trata-se de um estudo descritivo com dados obtidos de prontuários médicos. Foram estudadas variáveis relacionadas à neoplasia, ao portador, às toxicidades orais e ao tratamento oncológico, que foram analisadas descritivamente e o teste

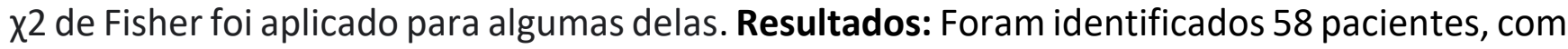
maioria do sexo feminino (53\%) e idade entre 3 e 5 anos (28\%), com média de 6,62 anos. A neoplasia maligna mais frequente foi a leucemia linfóide aguda (38\%), sendo a quimioterapia isolada a modalidade terapêutica mais empregada (67\%), especialmente com uso de citarabina (60\%) e vincristina (56\%). Vinte e três (40\%) das crianças e adolescentes exibiram alguma toxicidade oral durante a terapia oncológica, e por esta razão, $4(17,4 \%)$ tiveram o tratamento antineoplásico interrompido. A mucosite oral (70\%) foi a toxicidade mais comum, acometendo os lábios em 55\% e estando associada a dor em $64 \%$ dos casos. Dezoito (78,2\%) pacientes receberam tratamento para as toxicidades orais, sendo a nistatina prescrita em $88,9 \%$ dos casos. Ademais, observou-se associação significante entre status de sobrevida e local de residência ( $p=0.03)$. Conclusões: Dentre as 58 crianças e adolescentes estudadas, 40\% apresentou toxicidades orais e 4 tiveram o tratamento antineoplásico interrompido por esta razão. Desta forma, reforça-se a importância e a necessidade da atuação do cirurgião-dentista na equipe multiprofissional em oncologia.

Palavras chave: manifestações bucais; protocolos antineoplásicos; estomatite; equipe de assistência ao paciente.

\section{Introdução}

O câncer infantil corresponde a um grupo de várias doenças que têm em comum a proliferação descontrolada de células anormais e que pode ocorrer em qualquer local do organismo ${ }^{(1-3)}$. No Brasil, para cada ano do triênio 2020-2022, estima-se uma incidência de 8.460 novos casos ${ }^{(4)}$. Nesse contexto, a leucemia linfóide aguda (LLA) é a neoplasia mais frequente, seguida de tumores do sistema nervoso central (SNC) e linfomas ${ }^{(5-7)}$. 
O câncer infantil geralmente afeta as células do sistema sanguíneo e os tecidos de sustentação, que em geral, são constituídas de células indiferenciadas, resultando, na maioria dos casos, em uma melhor resposta aos métodos terapêuticos ${ }^{(4)}$. Dessa forma, associado aos progressos no tratamento oncológico, atualmente, se diagnosticados em estágios iniciais, cerca de $80 \%$ dos pacientes podem ser curados $(4,6,8)$.

As modalidades de tratamento das neoplasias malignas mais comumente utilizadas são a cirurgia, radioterapia (RT) e quimioterapia (QT), isoladas ou combinadas ${ }^{(9)}$. Nesse contexto, ademais das células neoplásicas, a QT e a RT atuam também sobre estruturas normais, principalmente as que sofrem maior número de divisão celular, como por exemplo a mucosa oral ${ }^{(10)}$. Por esta razão, muitos efeitos colaterais da terapia antineoplásica podem se manifestar na cavidade oral ${ }^{(10,11)}$. A incidência e a gravidade dessas toxicidades dependem de fatores como idade do indivíduo, tipo de neoplasia, modalidade terapêutica, tipo de quimioterápico, condição da cavidade oral anterior ao tratamento e nível de cuidado oral durante a terapia antineoplásica ${ }^{(5,6,12-14)}$.

As toxicidades orais agudas mais comuns são a mucosite, hemorragia, infecção, xerostomia, neurotoxicidade e disgeusia $(5,6,15,16)$. Tais toxicidades resultam em desconforto, dor, comprometimento da nutrição, redução da qualidade de vida, interrupções/atrasos/alterações nos tratamentos oncológicos, que consequentemente, afetam negativamente o prognóstico, e aumentam o tempo e os custos com hospitalização ${ }^{(17)}$. Desta forma, o objetivo deste estudo foi avaliar a prevalência de toxicidades orais decorrentes do tratamento oncológico em crianças e adolescentes atendidos no Hospital Estadual da Criança (HEC), no período de 2016 a 2017.

\section{Materiais e métodos}

Trata-se de um estudo observacional do tipo descritivo, retrospectivo, baseado em dados secundários dos registros de prontuários médicos. Pacientes portadores de outras doenças que não as neoplasias malignas ou de doença mental e indígenas não foram incluídos neste estudo.

O HEC está localizado na cidade de Feira de Santana, Bahia e oferta atendimento médico voltado para especialidades pediátricas de média e alta complexidade, incluindo a Oncologia. Esta pesquisa foi aprovada pelo comitê de Ética em Pesquisa da Universidade Estadual de Feira de Santana, sob parecer $n^{\circ} 035 / 2009$. 
Inicialmente foram identificados 65 prontuários de crianças e adolescentes atendidos na unidade de terapia oncológica do HEC, no período de 2016-2017. Após a aplicação dos critérios de elegibilidade, 58 casos compuseram a população final deste estudo.

A coleta de dados foi realizada através de formulário específico, validado por meio de um estudo piloto com $5 \%$ dos casos. As variáveis estudadas foram: a) sociodemográficas (idade, sexo, raça, local de residência, situação de trabalho do pai e da mãe, renda familiar mensal, abastecimento de energia, esgotamento sanitário e recolhimento de lixo); b) relacionadas à neoplasia (tipo de neoplasia maligna e localização) c) relacionadas às toxicidades orais (tipo de toxicidade, localização anatômica, sintomatologia e tratamento); d) relacionadas ao tratamento oncológico (modalidade terapêutica, medicamentos utilizados e status de sobrevida).

Os dados foram submetidos à análise descritiva, utilizando o programa SPSS (Statistical Package for the Social Sciences) na versão 17.0 (SPSS Inc., Chicago, IL, USA). Para as variáveis qualitativas foram utilizadas tabelas de frequência, com suas respectivas porcentagens, enquanto que para as variáveis quantitativas foram adotadas medidas descritivas, tais como média e desvio padrão. Ademais, foi realizado teste $\chi 2$ de Fisher para comparar as variáveis: tipo de neoplasia e modalidade terapêutica com o status de sobrevida; tipo de neoplasia e status de sobrevida com as condições socioedemográficas (local de residência, situação de trabalho do pai e da mãe, renda familiar mensal, abastecimento de energia, esgotamento sanitário e recolhimento de lixo). Para esta análise, foi adotado nível de significância de $5 \%$, onde $p \leq 0,05$ foi considerado significativo.

\section{Resultados}

Neste estudo, foram identificados 58 crianças e adolescentes. A maioria era do sexo feminino (53\%) e de cor não branca (100\%). A média de idade foi de $6,62( \pm 4,73)$ anos, com maior número de casos na faixa etária de 3-5 anos (28\%) (Tabela 1). As famílias eram provenientes sobretudo da zona urbana (66\%) e possuíam renda familiar mensal $\leq 1$ salário mínimo (60\%), estando $85 \%$ dos pais e $41 \%$ das mães ocupacionalmente ativos. Quanto às condições de moradia, 100\% possuía rede elétrica própria na residência, $56 \%$ contavam com tratamento de rede de esgoto na moradia e $86 \%$ com a coleta seletiva de lixo (Tabela 1 ). 
Tabela 1. Características sociodemográficas das crianças e adolescentes atendidas no HEC, no período de 2016-2017.

\begin{tabular}{|c|c|c|}
\hline Variáveis & $n$ & $\%$ \\
\hline \multicolumn{3}{|l|}{ Idade $(n=58)$} \\
\hline $0-2$ anos & 15 & $26 \%$ \\
\hline 3 -5 anos & 16 & $28 \%$ \\
\hline $6-8$ anos & 7 & $12 \%$ \\
\hline 9 -11 anos & 7 & $12 \%$ \\
\hline $12-14$ anos & 9 & $16 \%$ \\
\hline $15-17$ anos & 4 & $7 \%$ \\
\hline \multicolumn{3}{|l|}{$\operatorname{Sexo}(n=58)$} \\
\hline Feminino & 31 & $53 \%$ \\
\hline Masculino & 27 & $47 \%$ \\
\hline \multicolumn{3}{|l|}{ Raça/cor ( $n=58)$} \\
\hline Não Branco (Pardo/Negro) & 58 & $100 \%$ \\
\hline Branca & 0 & $0 \%$ \\
\hline \multicolumn{3}{|l|}{ Local de residência ( $n=58)$} \\
\hline Zona Urbana & 38 & $66 \%$ \\
\hline Zona Rural & 20 & $34 \%$ \\
\hline \multicolumn{3}{|c|}{ Situação de trabalho do paia ${ }^{a}(n=54)$} \\
\hline Ativo & 40 & $85 \%$ \\
\hline Inativo & 7 & $15 \%$ \\
\hline \multicolumn{3}{|c|}{ Situação de trabalho da mãe $e^{b}$} \\
\hline \multicolumn{3}{|l|}{$(n=47)$} \\
\hline Inativa & 32 & $59 \%$ \\
\hline Ativa & 22 & $41 \%$ \\
\hline \multicolumn{3}{|c|}{ Renda familiar mensalc $(n=48)$} \\
\hline$\leq$ de 1 salário & 29 & $60 \%$ \\
\hline Entre 1 e 3 salários & 15 & $31 \%$ \\
\hline$\geq 3$ salários & 4 & $8 \%$ \\
\hline \multicolumn{3}{|c|}{ Abastecimento de energia $^{d}(n=51)$} \\
\hline Elétrica própria & 51 & $100 \%$ \\
\hline Elétrica clandestina & 0 & $0 \%$ \\
\hline \multicolumn{3}{|c|}{ Esgotamento sanitário $^{e}(n=50)$} \\
\hline Tratamento de esgoto & 28 & $56 \%$ \\
\hline Fossa & 20 & $40 \%$ \\
\hline Céu aberto & 2 & $4 \%$ \\
\hline \multicolumn{3}{|l|}{ Recolhimento de lixo $(n=49)$} \\
\hline Sim & 42 & $86 \%$ \\
\hline Não & 7 & $14 \%$ \\
\hline
\end{tabular}

$a=4$ dados perdidos; $b=11$ dados perdidos; $c=10$ dados perdidos; $d=7$ dados perdidos; $e=8$ dados perdidos; $f=9$ dados perdidos. 
Dentre as neoplasias que acometeram a população estudada, a LLA foi a mais frequente (38\%), seguida dos tumores do SNC (19\%) e da leucemia mielóide aguda (LMA) (17\%). Quanto à localização, o sítio mais afetado foi a medula óssea (60\%) (Tabela 2). A QT isolada foi a modalidade terapêutica mais empregada (67\%), seguida da terapia combinada entre cirurgia e QT (16\%). A citarabina (60\%) e a vincristina (56\%) foram os quimioterápicos mais prescritos. Ao analisar o status de sobrevida, $41 \%$ dos indivíduos apresentaram remissão parcial da doença e $37 \%$ foram a óbito (Tabela 3 ).

Tabela 2. Tipo de neoplasia e localização que acometeram as crianças e adolescentes atendidas no HEC, período de 2016-2017.

\begin{tabular}{|c|c|c|}
\hline Variáveis & $n$ & $\%$ \\
\hline \multicolumn{3}{|l|}{ Tipo de neoplasia $^{a}(n=57)$} \\
\hline Leucemia Linfóide Aguda & 22 & $38 \%$ \\
\hline $\begin{array}{l}\text { Tumores do Sistema Nervoso } \\
\text { Central* }\end{array}$ & 11 & $19 \%$ \\
\hline Leucemia Mielóide Aguda & 10 & $17 \%$ \\
\hline Tumor Germinativo & 4 & $7 \%$ \\
\hline Sarcoma & 3 & $5 \%$ \\
\hline Retinoblastoma & 2 & $3 \%$ \\
\hline Outros & 2 & $3 \%$ \\
\hline Linfoma & 1 & $2 \%$ \\
\hline Tumor de Wilms & 1 & $2 \%$ \\
\hline Osteossarcoma & 1 & $2 \%$ \\
\hline \multicolumn{3}{|l|}{ Localização $^{b}(n=55)$} \\
\hline Medula Óssea & 33 & $60 \%$ \\
\hline Fígado & 3 & $5 \%$ \\
\hline Ovário & 3 & $5 \%$ \\
\hline Ponte & 2 & $4 \%$ \\
\hline Tronco cerebral & 2 & $4 \%$ \\
\hline Tronco Encefálico & 2 & $4 \%$ \\
\hline Fossa ilíaca & 1 & $2 \%$ \\
\hline Glândula Pineal & 1 & $2 \%$ \\
\hline Linha média & 1 & $2 \%$ \\
\hline Mandíbula & 1 & $2 \%$ \\
\hline Mediastino & 1 & $2 \%$ \\
\hline Mesencéfalo & 1 & $2 \%$ \\
\hline Órbita & 1 & $2 \%$ \\
\hline Região Glútea & 1 & $2 \%$ \\
\hline Pelve & 1 & $2 \%$ \\
\hline Vértebras C4 e C5 & 1 & $2 \%$ \\
\hline
\end{tabular}

$a=1$ dado perdido; $b=3$ dados perdidos.

*Tumores do Sistema Nervoso Central= 3 Tumores troncoencefálicos, 2 Ependimomas, 2 Gliomas, 1 Astrocitoma, 1 Craniofaringeoma, 1 Germinoma e 1 Tumor de células germinativas do sistema nervoso central. 
Tabela 3. Modalidade terapêutica, medicamento e status de sobrevida das crianças e adolescentes atendidas no HEC, período de 2016-2017.

\begin{tabular}{|c|c|c|}
\hline Variáveis & $n$ & $\%$ \\
\hline \multicolumn{3}{|l|}{ Modalidade terapêutica ( $n=58)$} \\
\hline Quimioterapia & 39 & $67 \%$ \\
\hline Cirurgia + Quimioterapia & 9 & $16 \%$ \\
\hline Cirurgia & 4 & $7 \%$ \\
\hline Quimioterapia + Radioterapia & 3 & $5 \%$ \\
\hline Nenhum tratamento & 2 & $3 \%$ \\
\hline Cirurgia + Quimioterapia + Radioterapia & 1 & $2 \%$ \\
\hline \multicolumn{3}{|l|}{ Medicamento $^{a}(n=52)$} \\
\hline Citarabina & 31 & $60 \%$ \\
\hline Vincristina & 29 & $56 \%$ \\
\hline Etoposide & 26 & $50 \%$ \\
\hline Metotrexate & 21 & $40 \%$ \\
\hline Daunorrubicina & 19 & $37 \%$ \\
\hline Mesna & 17 & $33 \%$ \\
\hline Tioguanina & 17 & $33 \%$ \\
\hline Asparaginase & 15 & $29 \%$ \\
\hline Ifosfamida & 15 & $29 \%$ \\
\hline Ciclofosfamida & 14 & $27 \%$ \\
\hline Marcaptopurina & 12 & $23 \%$ \\
\hline Cisplatina & 9 & $17 \%$ \\
\hline Idarrubicina & 9 & $17 \%$ \\
\hline Carboplatina & 8 & $15 \%$ \\
\hline Doxorrubicina & 6 & $12 \%$ \\
\hline Tretinoina & 5 & $10 \%$ \\
\hline Outros** & 7 & $13 \%$ \\
\hline \multicolumn{3}{|l|}{ Status de sobrevida ${ }^{b}(n=54)$} \\
\hline Remissão parcial & 22 & $41 \%$ \\
\hline Óbito & 20 & $37 \%$ \\
\hline Em tratamento & 7 & $13 \%$ \\
\hline Doença estável & 2 & $4 \%$ \\
\hline Sem evidência de doença & 1 & $2 \%$ \\
\hline Doença em progressão & 1 & $2 \%$ \\
\hline Fora de possibilidade terapêutica & 1 & $2 \%$ \\
\hline
\end{tabular}

$a=6$ dados perdidos e todos os pacientes foram tratados com mais de um medicamento;

$\mathrm{b}=4$ dados perdidos;

**Outros= 1 caso de Bleomicina, Dacarbazina, Dactinomicina, Imatinibe, Paclitaxel, Topotecano e Vimblastina, cada. 
Durante o tratamento antineoplásico, 23 (40\%) crianças e adolescentes exibiram alguma toxicidade oral e destes, $4(17,4 \%)$ tiveram o tratamento oncológico interrompido por esta razão. Estes 4 pacientes evoluíram com status de sobrevida: doença estável, remissão parcial, retomada do tratamento e um não possuía informação sobre esta variável. A mucosite oral esteve presente em $50 \%$ dos casos e a candidíase em $34 \%$, acometendo sobretudo os lábios (55\%), mucosa jugal (36\%) e língua (32\%). Os principais sintomas associados às toxicidades orais foram a dor (64\%) e a disfagia (45\%) (Tabela 4). Dentre eles, 18 (78\%) realizaram tratamento para as toxicidades orais, sendo a nistatina o medicamento mais prescrito $(88,9 \%)$, de forma isolada ou associada a outras terapêuticas.

Tabela 4. Presença, tipos, localização, sintomatologia e tratamento para toxicidades orais das crianças e adolescentes atendidas no HEC, no período de 2016-2017.

\begin{tabular}{|c|c|c|}
\hline Variáveis & $n$ & $\%$ \\
\hline \multicolumn{3}{|c|}{ Presença de toxicidade oral $(n=58)$} \\
\hline Não & 35 & $60 \%$ \\
\hline Sim & 23 & $40 \%$ \\
\hline \multicolumn{3}{|c|}{ Tipo de toxicidade oral ${ }^{a}(n=23)$} \\
\hline Mucosite oral & 16 & $70 \%$ \\
\hline Candidíase & 11 & $48 \%$ \\
\hline Outra & 2 & $9 \%$ \\
\hline Xerostomia & 1 & $4 \%$ \\
\hline Herpes & 1 & $4 \%$ \\
\hline Trismo & 1 & $4 \%$ \\
\hline \multicolumn{3}{|c|}{ Localização $^{b}(n=22)$} \\
\hline Lábios & 12 & $55 \%$ \\
\hline Mucosa jugal & 8 & $36 \%$ \\
\hline Língua & 7 & $32 \%$ \\
\hline Gengiva & 3 & $14 \%$ \\
\hline Não especificada & 3 & $14 \%$ \\
\hline Assoalho bucal & 1 & $5 \%$ \\
\hline Palato duro & 1 & $5 \%$ \\
\hline Palato mole & 1 & $5 \%$ \\
\hline \multicolumn{3}{|c|}{ Sintoma associado à toxicidade oral $(n=11)$} \\
\hline Dor & 7 & $64 \%$ \\
\hline Disfagia & 5 & $45 \%$ \\
\hline Ardor & 1 & $9 \%$ \\
\hline Outra & 2 & $18 \%$ \\
\hline
\end{tabular}

$\mathrm{a}=7$ pacientes apresentaram 2 toxicidades associadas e 1 apresentou 3; $\mathrm{b}=1$ dado perdido e 10 pacientes apresentaram acometimento de mais de uma localização; $\mathrm{C}=12$ dados perdidos e 4 pacientes apresentaram 2 sintomas. 
Ao analisar o status de sobrevida com o tipo de neoplasia $(p=0.432)$ e a modalidade terapêutica $(p=0.08)$, não houve associação entre as variáveis. A análise do tipo de neoplasia em relação às condições sociodemográficas também não resultou em associações estatisticamente significantes: local de residência $(p=0.74)$; situação trabalho pai $(p=0.94)$ e da mãe $(p=0.21)$; renda familiar mensal $(p=0.49)$; abastecimento de energia $(p=0.76)$; esgotamento sanitário $(p=$ 0.92); e recolhimento de lixo $(p=0.30)$. Por fim, a análise do status de sobrevida e as variáveis sociodemográficas evidenciou associação estatisticamente significante para o local de residência $(p=0.03)$, enquanto que para as demais variáveis não houve associação: situação trabalho pai $(p=0.61)$ e da mãe $(p=0.70)$; renda familiar mensal $(p=0.06)$; abastecimento de energia (não foi possível calcular); esgotamento sanitário $(p=0.31)$; e recolhimento de lixo $(p=0.07)$.

\section{Discussão}

As neoplasias malignas em crianças e adolescentes, em países dos diferentes continentes, tende a acometer com maior frequência o sexo masculino, sobretudo no primeiro ano de vida, ou entre o segundo e terceiro anos ${ }^{(18-20)}$. No presente estudo observou-se maior número de casos em indivíduos do sexo feminino (53\%) e com idade entre 3 e 5 anos (28\%) seguida de 0 a 2 anos (26\%), entretanto, deve-se ressaltar que essas diferenças foram pequenas e considerar o número reduzido de indivíduos estudados.

Os principais fatores de risco associados ao desenvolvimento do câncer em crianças e adolescentes são os fatores genéticos herdados ou mutações adquiridas de causa incerta, ademais dos fatores exógenos como agentes físicos, químicos e biológicos ${ }^{(4,21)}$. Apesar da existência de potenciais fatores de risco por exposição intrauterina da criança, não existem evidências científicas suficientes que suportem a associação entre o câncer infantil e os fatores ambientais. Dessa forma, a prevenção do câncer infantil ainda é um desafio e a ênfase atual na abordagem a esse tipo de câncer deve ser dada ao diagnóstico precoce e encaminhamento imediato para um tratamento oportuno e de qualidade, que possibilite maiores taxas de cura ${ }^{(21)}$.

No presente estudo, observou-se associação estatisticamente significante entre o status de sobrevida e o local de residência. Nessa perspectiva, outros autores já haviam relatado que condições socioeconômicas desfavoráveis podem ser agravantes às dificuldades já impostas pelo câncer ${ }^{(22)}$, como influenciar no acesso a serviços de diagnóstico, seja por falta de conhecimento ou pela distância. Os indivíduos deste estudo residiam sobretudo em zona urbana, com renda mensal de no máximo um salário mínimo e com acesso a saneamento 
básico. Ademais, observou-se que a maioria dos pais eram ocupacionalmente ativos, enquanto as mães eram majoritariamente inativas. Esse quadro provavelmente está relacionado ao fato da mãe ser a principal cuidadora dos filhos, ou seja, são elas que costumam levar as crianças às consultas e realizar os demais cuidados ${ }^{(2)}$, enquanto que os pais tornam-se os provedores da renda. Além disso, Cheng et al. (2008) relatam que um estado socioeconômico desfavorecido está geralmente relacionado a uma condição bucal mais desfavorável e a uma maior prevalência de cáries, o que aumenta o risco de complicações durante a terapia oncológica (23).

No que diz respeito ao tipo, a LLA é considerada a neoplasia mais frequente em crianças e adolescentes (20), seguida dos tumores do SNC, corroborando nossos resultados. Outras neoplasias malignas que acometem frequentemente esta população são o neuroblastoma, tumor de Wilms, retinoblastoma, tumor germinativo e sarcomas, que também foram encontradas no nosso estudo ${ }^{(5-7)}$. Além disso, destacamos a presença de um caso de osteossarcoma em mandíbula, considerado um tumor raro tanto pelo acometimento da região de cabeça e pescoço, quanto pela faixa etária ${ }^{(21,24)}$.

De forma geral, o tratamento das neoplasias pode abranger a cirurgia, a RT e a QT, sendo a QT empregada em cerca de $70 \%$ dos casos ${ }^{(18,25)}$, corroborando nossos achados. Os quimioterápicos mais prescritos para os pacientes do presente estudo foram a citarabina e a vincristina, que são drogas integrantes dos protocolos empregados para o tratamento da LLA ${ }^{(6)}$, neoplasia mais frequente no nosso estudo. Dentre os pacientes analisados, $41 \%$ apresentaram remissão parcial da doença e $37 \%$ evoluíram para óbito. Nesse sentido, é importante ressaltar que apesar do progresso nos tratamentos oncológicos e o maior acesso oferecido à população, o câncer ainda é considerado a principal causa de morte por doença entre crianças e adolescentes no Brasil (21).

Apesar das evoluções, a RT e a QT atuam destruindo ou inibindo o crescimento de células com elevado índice mitótico, sem diferenciar células tumorais das saudáveis causando, assim, toxicidades em outras partes do organismo, como na cavidade oral ${ }^{(18)}$. Neste contexto, as crianças tendem a ser três vezes mais susceptíveis a essas toxicidades e dentre elas, as mais incidentes são a mucosite, infecções oportunistas, sangramento, xerostomia, dor, alterações de sabor, neuropatias e trismo ${ }^{(20)}$, corroborando nossos achados.

De forma geral, os resultados do nosso estudo a respeito das toxicidades orais corroboram a literatura. A mucosite possui incidência variável entre 10 e $100 \%$ nas diferentes modalidades terapêuticas e especificamente em pacientes tratados com QT, acomete cerca de $40 \%$ dos 
indivíduos ${ }^{(18,20)}$. Dentre os quimioterápicos, o metotrexato, a ciclofosfamida, doxorrubicina, bleomicina, vimblastina, vincristina e o 5-fluorouracil são considerados estomatotóxicos, e estão mais relacionados com o surgimento da mucosite oral ${ }^{(5,6)}$. As lesões podem variar entre eritema e úlceras, que acometem sobretudo regiões de epitélio não-queratinizado, como mucosa jugal, assoalho de boca e ventre de língua. Entratenado, nos casos mais graves as superfícies queratinizadas também podem ser afetadas (26). Dessa forma, a perda de continuidade do epitélio, associada a imunossupressão e a redução do fluxo salivar, contribuem para o aparecimento de infecções oportunistas ${ }^{(27-30)}$. Nesse contexto, a candidíase é a infecção fúngica mais frequente ${ }^{(20)}$.

Segundo a Multinational Association of Supportive Care in Cancer, apesar de ainda não ser possível estabelecer um guideline, estudos recentes demonstraram eficácia da terapia de fotobiomodulação no tratamento da mucosite oral em pacientes pediátricos ${ }^{(31)}$. Entretanto, em muitos centros essa terapia não pode ser empregada pela ausência do equipamento ou de profissionais habilitados. Desta forma, o manejo da mucosite oral passa a ser paliativo, por meio do controle dos sintomas através de anestésicos tópicos e analgésicos sistêmicos. Além disso, atua-se na prevenção de infecções secundárias através de instrução de higiene oral, prescrição de antifúngicos tópicos e enxaguatórios à base de clorexidina a $0,12 \%$ e no tratamento específico para os quadros de infecção já instaladas $(20,32,33)$.

Diante da presença de lesões de mucosite e infecções oportunistas, os pacientes apresentam quadro clínico que envolve sintomas de dor, queimação e disfagia ${ }^{(34)}$. Este quadro pode acarretar em uma nutrição inadequada, conduzindo o paciente a perda de peso, anorexia, caquexia e desidratação ${ }^{(24)}$. Dessa forma, as toxicidades orais podem resultar em atrasos na administração, limitações de dosagens dos quimioterápicos, aumento do tempo de hospitalizações e dos custos do tratamento ${ }^{(17,20,35)}$, como pôde ser observado em $17,4 \%$ dos pacientes deste estudo. Nesse sentido, a atuação do cirurgião-dentista durante a terapia oncológica auxilia na continuidade do tratamento, com melhor qualidade de vida, e consequentemente, contribui para um melhor prognóstico e sobrevida do indivíduo ${ }^{(36)}$.

Dentre os sobreviventes do câncer infantil, aproximadamente 75\% terão um problema crônico de saúde resultante da terapia oncológica ${ }^{(37)}$. Nesse contexto, o cirurgião-dentista deve estar atento para diagnosticar e manejar as toxicidades orais tardias através de estratégias individualizadas. Especificamente para pacientes submetidos a RT em região de cabeça e pescoço ou QT associada a irradiação de corpo inteiro antes de 5-6 anos, costumam ser observadas alterações no desenvolvimento cranio facial e alterações na formação dentária ${ }^{(20)}$. 
Devido às limitações próprias deste modelo de estudo, não se permite fazer associações de causalidade entre as variáveis estudadas, uma vez que trata-se de um estudo descritivo. Desta forma, são necessários novos estudos longitudinais, que permitam um melhor entendimento a respeito dos fatores associados às neoplasias e toxicidades orais em crianças e adolescentes. Complementarmente, por ser um estudo baseado em dados secundários dos prontuários médicos, ressalta-se a importância do correto e completo preenchimento destes documentos.

Apesar disto, os resultados do presente estudo evidenciam a importância da inserção do cirurgião-dentista na equipe multiprofissional, a fim de atuar no preparo odontológico para o tratamento oncológico, bem como diagnosticar e manejar as toxicidades orais agudas e crônicas. Ademais, ressalta-se a necessidade do desenvolvimento de políticas públicas específicas voltadas para a saúde bucal deste grupo específico de pacientes.

\section{Conclusões}

Dentre as 58 crianças e adolescentes que receberam tratamento oncológico no HEC no período 2016 e 2017, 40\% apresentou toxicidades orais e 4 tiveram o tratamento antineoplásico interrompido por esta razão. A mucosite e a candidíase foram as mais comuns, acometendo principalmente os lábios e associadas a dor. A nistatina foi o medicamento mais utilizado para o manejo. A presença destas toxicidades orais indica a necessidade de um cirurgião-dentista na equipe multiprofissional de tratamento do paciente oncológico infanto-juvenil. Consultas prétratamento, adequação do meio bucal e acompanhamento odontológico sistemático são primordiais para prevenção, controle e tratamento destas toxicidades.

\section{Referências}

1. Amador DD, Gomes IP, Coutinho SED, Costa TNA, Collet N. Concepção dos enfermeiros acerca da capacitação no cuidado à criança com câncer. Texto e Context Enferm. 2011;20(1):94-101.

2. Nascimento LC, Rocha SMM, Hayes VH, Lima RAG. Crianças com câncer e suas famílias. Rev Esc Enferm USP. 2005;39(4):469-74.

3. Instituto Nacional de Câncer (Brasil). Câncer na criança e no adolescente no Brasil /Dados dos Registros de Base Populacional. Journal of Chemical Information and Modeling. Rio de Janeiro: Instituto Nacional de Câncer; 2008. p. 220.

4. INCA/Ministério da Saúde. Estimativa 2020: incidência de câncer no Brasil / Instituto Nacional de Câncer José Alencar Gomes da Silva. Rio de Janeiro: INCA; 2019. 
5. Ponce-Torres E, Ruíz-Rodríguez MDS, Alejo-González F, Hernández-Sierra JF, De PozosGuillén AD. Oral manifestations in pediatric patients receiving chemotherapy for acute Iymphoblastic leukemia. J Clin Pediatr Dent. 2010;34(3):275-9.

6. Subramaniam P, Girish Babu KL, Nagarathna J. Oral manifestations in acute lymphoblastic leukemic children under chemotherapy. J Clin Pediatr Dent. 2008;32(4):319-24.

7. Soares AF, de Aquin ARL, de Carvalh CHP, Nonaka CFW, Almeida D, Pereira Pinto L. Frequency of oral mucositis and microbiological analysis in children with acute lymphoblastic leukemia treated with $0.12 \%$ chlorhexidine gluconate. Braz Dent J. 2011;22(4):312-6.

8. Avşar A, Elli M, Darka Ö, Pinarli G. Long-term effects of chemotherapy on caries formation, dental development, and salivary factors in childhood cancer survivors. Oral Surgery, Oral Med Oral Pathol Oral Radiol Endodontology. 2007;104(6):781-9.

9. Saletta F, Seng MS, Lau LMS. Advances in paediatric cancer treatment. Transl Pediatr. 2014;3(2):156-15682.

10. Glenny AM, Gibson F, Auld E, Coulson S, Clarkson JE, Craig J V., et al. A survey of current practice with regard to oral care for children being treated for cancer. Eur J Cancer. 2004;40(8):1217-24.

11. Peterson DE. Oral problems in supportive care: No longer an orphan topic? Support Care Cancer. 2000;8(5):347-8.

12. El -Housseiny AA, Saleh SM, El-Masry AA, Allam AA. Assessment of oral complications in children receiving chemotherapy. J Clin Pediatr Dent. 2007;31(4):267-73.

13. Xavier AM, Hegde AM. Preventive protocols and oral management in childhood leukemia - the pediatric specialist's role. Asian Pacific J Cancer Prev. 2010;11(1):39-43.

14. Minicucci EM, Lopes LF, Crocci AJ. Dental abnormalities in children after chemotherapy treatment for acute lymphoid leukemia. Leuk Res. 2003;27(1):45-50.

15. Eilers J, Million R. Clinical update: Prevention and management of oral mucositis in patients with cancer. Semin Oncol Nurs. 2011;27(4):e1-16. 
16. Cauwels RGEC, Martens LC. Low level laser therapy in oral mucositis: A pilot study. Eur Arch Paediatr Dent. 2011;12(2):118-23.

17. Lalla R V., Saunders DP, Peterson DE. Chemotherapy or Radiation-Induced Oral Mucositis. Dent Clin North Am. 2014;58(2):341-9.

18. Peres P, Queiroz AM de, Moreira MR, Faquim JP da S, Ferrari MACM. Odontopediatria aplicada ao Câncer Infantil - Manifestações Clínicas e Protocolo de Atendimento. JMPHC | J Manag Prim Heal Care | ISSN 2179-6750. 2013;4(3):199.

19. Braga PE, Latorre M do RD de O, Curado MP. Câncer na infância: análise comparativa da incidência, mortalidade e sobrevida em Goiânia (Brasil) e outros países. Cad Saude Publica. 2002;18(1):33-44.

20. Hong $\mathrm{CH}$, daFonseca $\mathrm{M}$. Considerations in the Pediatric Population with Cancer. Dent Clin North Am. 2008;52(1):155-81.

21. Ministério da Saúde. Protocolo de Diagnóstico Precoce do Câncer Pediátrico. Ministério da Saúde. Brasília: Ministério da Saúde do Brasil; 2017.

22. Nilkece S, Araújo M, Maria E, Rangel L, Celia A, Melo M, et al. Oral mucositis: sociodemographic analysis in cancer patients. ReOnFacema. 2015;1(1):3-8.

23. Cheng KKF, Goggins WB, Lee VWS, Thompson DR. Risk factors for oral mucositis in children undergoing chemotherapy: A matched case-control study. Oral Oncol. 2008;44(11):1019-25.

24. Benoit MMK, Vargas SO, Bhattacharyya N, McGill TA, Robson CD, Ferraro N, et al. The presentation and management of mandibular tumors in the pediatric population. Laryngoscope. 2013;123(8):2035-42.

25. Barbosa AM, Ribeiro DM, Caldo-Teixeira AS. Conhecimentos e práticas em saúde bucal com crianças hospitalizadas com câncer. Cien Saude Colet. 2010;15(suppl 1):1113-22.

26. Fadda G, Campus G, Lugliè PF. Risk factors for oral mucositis in paediatric oncology patients receiving alkylant chemotherapy. BMC Oral Health. 2006;6:1-8.

27. Lino MDM da C, de Carvalho FB, de Oliveira LR, Magalhães EB, Pinheiro ALB, Ramalho LMP. Laser phototherapy as a treatment for radiotherapy-induced oral mucositis. Braz Dent J. 2011;22(2):162-5. 
28. de Lima AG, Antequera R, Peres MPS de M, Snitcosky IML, Federico MHH, Villar RC. Efficacy of low-level laser therapy and aluminum hydroxide in patients with chemotherapy and radiotherapy-induced oral mucositis. Braz Dent J. 2010;21(3):18692.

29. Javed F, Utreja A, Bello Correa FO, Al-Askar M, Hudieb M, Qayyum F, et al. Oral health status in children with acute lymphoblastic leukemia. Crit Rev Oncol Hematol. 2012;83(3):303-9.

30. Albuquerque RA, Morais VLL, Sobral APV. Protocolo de atendimento odontológico a pacientes oncológicos pediátricos - revisão de literatura. Rev Odontol da UNESP. 2007;36(3):275-80.

31. Zadik Y, Arany PR, Fregnani ER, Bossi P, Antunes HS. Systematic review of photobiomodulation for the management of oral mucositis in cancer patients and clinical practice guidelines. Support Care Cancer. 2019.

32. Javed F, Al-Hezaimi K, Romanos GE. Role of dentifrices with essential oil formulations in periodontal healing. Am J Med Sci. 2012;343(5):411-7.

33. Pereira Pinto L, de Souza LB, Gordón-Núñez MA, Soares RC, de Brito Costa EMM, de Aquino ARL, et al. Prevention of oral lesions in children with acute lymphoblastic leukemia. Int J Pediatr Otorhinolaryngol. 2006;70(11):1847-51.

34. Pioch T, D. G, Staehle HJ. An experimental study of the stability of irradiated teeth in the region of the dentinoenamel junction. Endod Dent Traumatol. 1992;8:241-4.

35. Sasada INV, Cancino CMH, Petersen RC, Hellwig I, Dillenburg CS. Prevenção de intercorrências estomatológicas em oncologia pediátrica. Rev da Fac Odontol - UPF. 2015;20(1):105-9.

36. Alves LDB, Menezes AC dos S, da Costa AMD, Heimlich FV, Goldemberg DC, Antunes HS. Panorama atual dos programas de Residência Multiprofissional em Oncologia para cirurgiões-dentistas do Brasil. Rev Bras Cancerol. 2020;66(3):1-9.

37. Dickerman JD. The late effects of childhood cancer therapy. Pediatrics. 2007;119(3):554-68. 